\title{
Targeting P-Selectin Adhesion Molecule in Molecular Imaging: P-Selectin Expression as a Valuable Imaging Biomarker of Inflammation in Cardiovascular Disease
}

\author{
Lydia A. Perkins, Carolyn J. Anderson, and Enrico M. Novelli \\ Department of Medicine, University of Pittsburgh, Pittsburgh, Pennsylvania
}

\begin{abstract}
P-selectin is an adhesion molecule translocated to the surface of endothelial cells and platelets under inflammatory stimuli, and its potential as a biomarker in inflammatory conditions has driven preclinical studies to investigate its application for molecular imaging of inflammation. Clinical imaging of P-selectin expression for disease characterization could have an important role in stratifying patients and determining treatment strategies. The objective of this review is to outline the role of P-selectin in cardiovascular inflammatory conditions and its translation as an early inflammatory biomarker for several molecular imaging modalities for diagnostic purposes and therapeutic planning.
\end{abstract}

Key Words: P-selectin; molecular imaging; inflammation; inflammatory imaging biomarker

J Nucl Med 2019; 60:1691-1697

DOI: 10.2967/jnumed.118.225169

I nflammation is a critical process at the root of many pathologic conditions involving essentially every organ system in the body and contributing to many disease states. As examples, cardiovascular diseases, cancer, autoimmune diseases, benign hematologic diseases (e.g., sickle cell disease), and neurologic diseases are all implicated, with inflammation at various stages induced by different biologic mechanisms of action. Without prompt treatment at disease onset, inflammatory pathologies can lead to extensive and irreversible damage, increased pain, and a poorer prognosis (1). Reliably assessing inflammation early and throughout the disease course requires sensitive diagnostic methods (2). Noninvasive molecular imaging modalities, including ultrasound, MRI, SPECT, and PET, have been used as supportive diagnostic tools for physicians in evaluating inflammation (3). Traditional imaging techniques can be used to assess tissue morphology changes due to inflammation, whereas nuclear medicine-based imaging methods - PET and SPECT — are able to functionally and molecularly measure inflammation with high sensitivity.

Received Aug. 2, 2019; revision accepted Oct. 4, 2019

For correspondence or reprints contact: Enrico M. Novelli, E1257 BST, 200

Lothrop St., Pittsburgh, PA 15261.

E-mail: novellie@upmc.edu

Published online Oct. 10, 2019.

COPYRIGHT (c) 2019 by the Society of Nuclear Medicine and Molecular Imaging.
Nonetheless, clinical nuclear agents currently used for inflammation imaging, such as ${ }^{18} \mathrm{~F}-\mathrm{FDG}$, which accumulates in tissues with increased cellular glycolytic activity, are limited in scope by high background levels or nonspecific uptake that can complicate analysis and interpretation $(4,5)$. With more recent preclinical advances, new contrast agents developed for MRI and ultrasound are emerging as strong contenders to image at the molecular level for diagnosing and monitoring inflammation (6,7). The clinical demand for sensitive molecular imaging agents for early and reliable characterization of inflammation has charged preclinical research with developing selective targeting strategies using PET, SPECT, ultrasound, and MRI.

Researchers are investigating hallmark biomarkers expressed in inflammation to develop targeted agents for imaging specific aspects of inflammation. Among disease biomarker candidates, preclinical research has focused on agents that bind cell adhesion molecules to characterize inflammatory responses across diseases. A recent review by Lee et al. discusses targeting of cell adhesion molecules in chronic inflammatory diseases, focusing on preclinical PET/SPECT imaging of integrins, the immunoglobulin superfamily (vascular cell adhesion molecule 1, intercellular adhesion molecule 1), and selectins (2).

The adhesion molecule, P-selectin, has been the focus of intense investigation as a molecular imaging target for early inflammatory disease states and shows promise in assisting clinical disease staging, treatment planning, and monitoring. The focus of this review is to highlight the pivotal role P-selectin plays among inflammatory states and its current preclinical application as a biomarker of inflammation across molecular imaging platforms.

\section{P-SELECTIN INVOLVEMENT IN INFLAMMATORY DISEASES: THE ROLE OF HYPERADHESION}

Although disease-specific factors can elicit inflammation and produce diverse physiologic manifestations, the activation of the vascular endothelium remains a crucial part of the inflammatory process across pathologic states. The inflammatory response is initiated by cytokine signaling that activates the local blood vessel endothelium for recruitment, adhesion, and diapedesis of leukocytes. 
Leukocyte attachment to the vessel endothelium involves a regulated succession of specific adhesion molecules expressed at the cell surface to facilitate the multistep adhesion process of leukocyte tethering, rolling, and ultimately adherence and diapedesis. The activated endothelium mediates the first "catching" contacts with leukocytes through triggering cell surface expression of a cell adhesion molecule, P-selectin. Within minutes, readily available pools of $\mathrm{P}$-selectin are trafficked to the endothelial cell surface from cytoplasmic storage granules. At the surface, P-selectin binds to its counterligand, P-selectin glycoprotein ligand 1 (PSGL-1), which is constitutively expressed on leukocytes. The P-selectin/ PSGL-1 transient interactions slow leukocytes to roll along the vessel endothelium, allowing for stronger attachments to form with subsequently expressed or activated adhesion molecules (Figs. 1A and 1B) (8).

Aberrant or persistent vascular endothelial activation caused by inflammatory triggers can result in overexpression of adhesion molecules and in turn increase leukocyte attachment and infiltration, which can damage the vasculature and

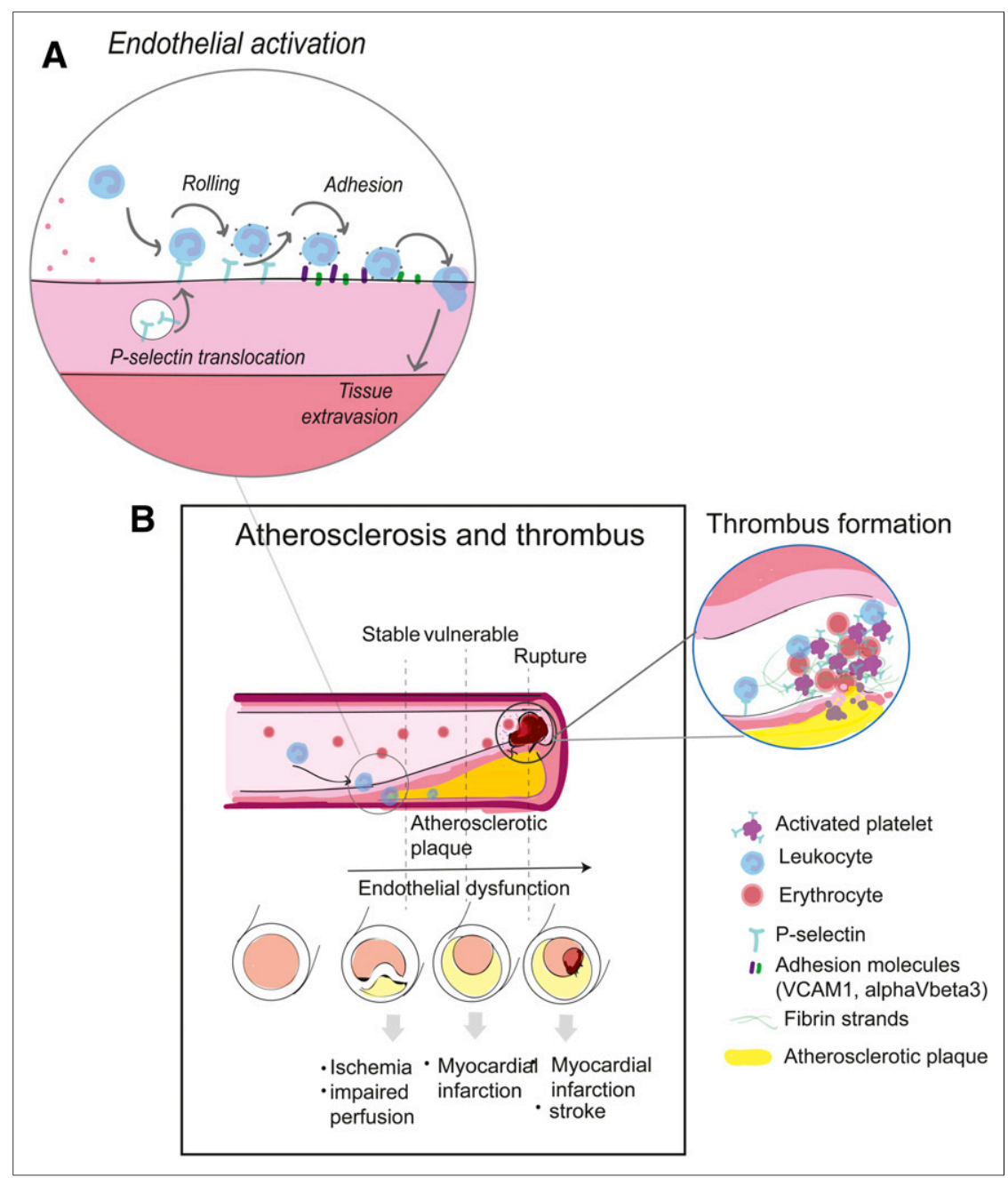

FIGURE 1. P-selectin role in endothelial activation (A) and in atherosclerosis and thrombus (B). localized tissue (9). Damage to the vessel endothelium through destructive inflammation or injury can additionally activate local platelets to express P-selectin, facilitating aggregation and adhesive interactions between platelets, leukocytes, and endothelium. Like endothelial cells, platelets also have intracellular storage pools of P-selectin in the $\alpha$-granules that are quickly translocated to the platelet cell surface on specific inflammatory stimulation (10). The platelet expression of P-selectin promotes endothelial adhesive crosslinks between leukocytes and the endothelium, contributing to an upregulated release of proinflammatory cytokines and increased adhesion to the vessel endothelium $(10,11)$.

P-selectin expression by activated endothelial cells and activated platelets has been shown to be a substantial feature of inflammation-related pathologic states. An extensive review by Kappelmayer and Nagy outlined the detailed involvement of P-selectin and its ligand in inflammation by modulating leukocyte recruitment, thrombus development, and cancer progression (12). The increased surface expression of P-selectin during inflammation, especially as it is involved in the initial leukocyte endothelial adhesion, makes it an attractive and sensitive biomarker to reveal early pathologic changes and monitor disease treatment.

\section{P-SELECTIN TARGETING}

The potential of P-selectin as an imaging biomarker and molecular target for therapies has been investigated through targeted strategies using antibodies and polysaccharide ligand mimics. The lectin domain of P-selectin (Fig. 2A) has high binding affinity toward the oligosaccharide, sialyl Lewis ${ }^{\mathrm{X}}$ (sLe ${ }^{\mathrm{x}}$ ), on PSGL-1 (Fig. 2B). Polysaccharides that harbor sLe ${ }^{\mathrm{x}}$ structures or are $\mathrm{sLe}^{\mathrm{x}}$ mimics can also be recognized by P-selectin. This ligand structure can be harnessed to target imaging agents to P-selectin. Recently, fucoidan, an $\mathrm{sLe}^{\mathrm{x}}$ mimic derived from algae, has been demonstrated to be a low-cost and effective targeting reagent for P-selectin in SPECT, MRI, and ultrasound techniques (13). A review by Wang et al. discussed the biologic activity of fucoidan and its beneficial effect as an antitumor, antioxidant, anticoagulant, antithrombotic, immunoregulatory, antiviral, and antiinflammatory therapeutic. However, fucoidan's pharmacokinetics have not been investigated, as variances in its structure, molecular weight, extraction, and algae source are largely uncharacterized (14). Despite its limited characterization, the use of fucoidan in preclinical studies as a 


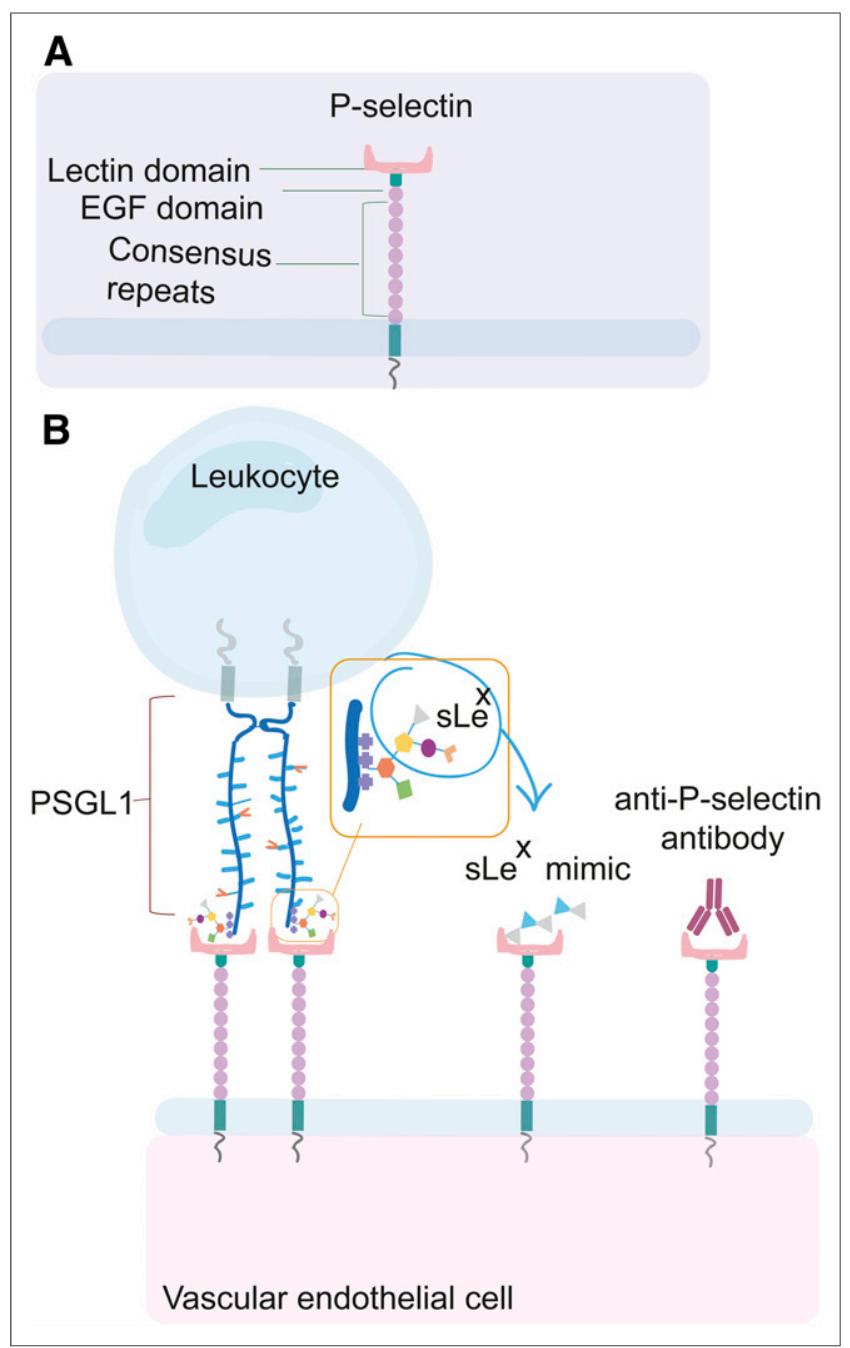

FIGURE 2. P-selectin and its ligands. (A) P-selectin structure. (B) P-selectin binds its natural counterligand PSGL1, which is expressed on leukocytes. P-selectin can bind to different polysaccharides that are $s \mathrm{Se}^{\mathrm{x}}$ mimics. Anti-P-selectin antibodies can also target the active binding site or other sites on P-selectin. EGF $=$ epidermal growth factor.

therapeutic and targeting imaging agent is increasing, with promising results.

\section{MOLECULAR IMAGING OF P-SELECTIN IN PRECLINICAL STUDIES}

The development of targeted P-selectin molecular imaging agents has been centered largely on assessing inflammation associated with cardiovascular disease, which remains a leading cause of death despite decades of progress in the prevention of stroke and myocardial infarction $(15,16)$. By developing an early detection strategy, particularly for those at increased risk, timely treatment can be delivered to improve patient morbidity and mortality. Cardiovascular disease commonly manifests by formation of plaques along the arterial vascular wall (atherosclerosis). This condition develops slowly over time and can gradually constrict blood flow through the arteries, causing life-threatening complications even in the absence of any easily discernable symptoms. The process of atherosclerotic plaque buildup is complex and progresses in distinct steps. Trauma, inflammation, and many other factors can trigger or alter the course of the disease (17). A recent review discusses a new perspective on the importance of applying selective imaging strategies in evaluating characteristic stages of atherogenesis and outlines the current clinical imaging modalities that may be appropriate at each stage (18). Available detection methods for evaluating early stages, however, lack the necessary accuracy to assess the molecular processes of plaque formation, vulnerability, and rupture $(17,18)$.

The initial step of atherosclerosis-endothelial activation along the vessel wall-is followed by increased expression of adhesive molecules for leukocyte recruitment in areas of injury from lipid accumulation or hypertensive force $(18,19)$. Leukocytes undergo intravasation into the inner layer of the vessel alongside myocytes for tissue repair, leading to monocytederived foam cells within the plaque and a fibrous cap, respectively. Inflamed plaques can rupture, causing tissue ischemia and triggering thrombus formation, which clinically may result in stroke or myocardial infarction, among other complications (Fig. 1B) (20). It is critical to identify and evaluate atherosclerotic plaques before rupture to prevent severe cardiovascular disease; however, most active lesions remain undetected until rupture and thrombosis occur. A link between thrombogenesis and inflammation has been proposed, with P-selectin expression augmenting thrombotic risk, making P-selectin an increasingly attractive biomarker for atherothrombotic disease (21).

Inflamed plaques express high levels of P-selectin, which could potentially represent a biomarker of active plaque bioactivity and rupture risk. In addition, P-selectin also becomes more prominent during thrombus formation, with platelet activation facilitating platelet-leukocyte aggregation (22).

A study by $\mathrm{Li}$ et al. used ${ }^{68} \mathrm{Ga}$-labeled fucoidan for Pselectin PET imaging of vulnerable atherosclerotic plaques by PET/MRI in an early atherogenesis mouse model, demonstrating the first use of ${ }^{68} \mathrm{Ga}$-fucoidan to image P-selectin expression by PET via an efficient and reproducible tracer synthesis protocol (22). Li et al. used apolipoprotein E-deficient mice and measured P-selectin expression with PET after $60 \mathrm{~min}$ of tracer uptake, reporting a plaque-to-background SUV ratio of $5.1 \pm 0.08$. The study showed clear uptake at the vascular wall at many aortal segments (Fig. 3) and demonstrated the potential of P-selectin imaging in early inflammatory atherosclerotic plaques.

Publications reporting on ultrasound molecular imaging of inflammation have been increasing. A review by AbouElkacem, et al. cited several examples of research studies using microbubbles targeting P-selectin or dual selectin (P\&Eselectin) for myocardial ischemia, atherosclerosis, and inflammatory bowel disease (7). Herein, we will review those targeting P-selectin.

One of the ultrasound imaging studies described a polymer microcapsule functionalized with fucoidan to target P-selectin 


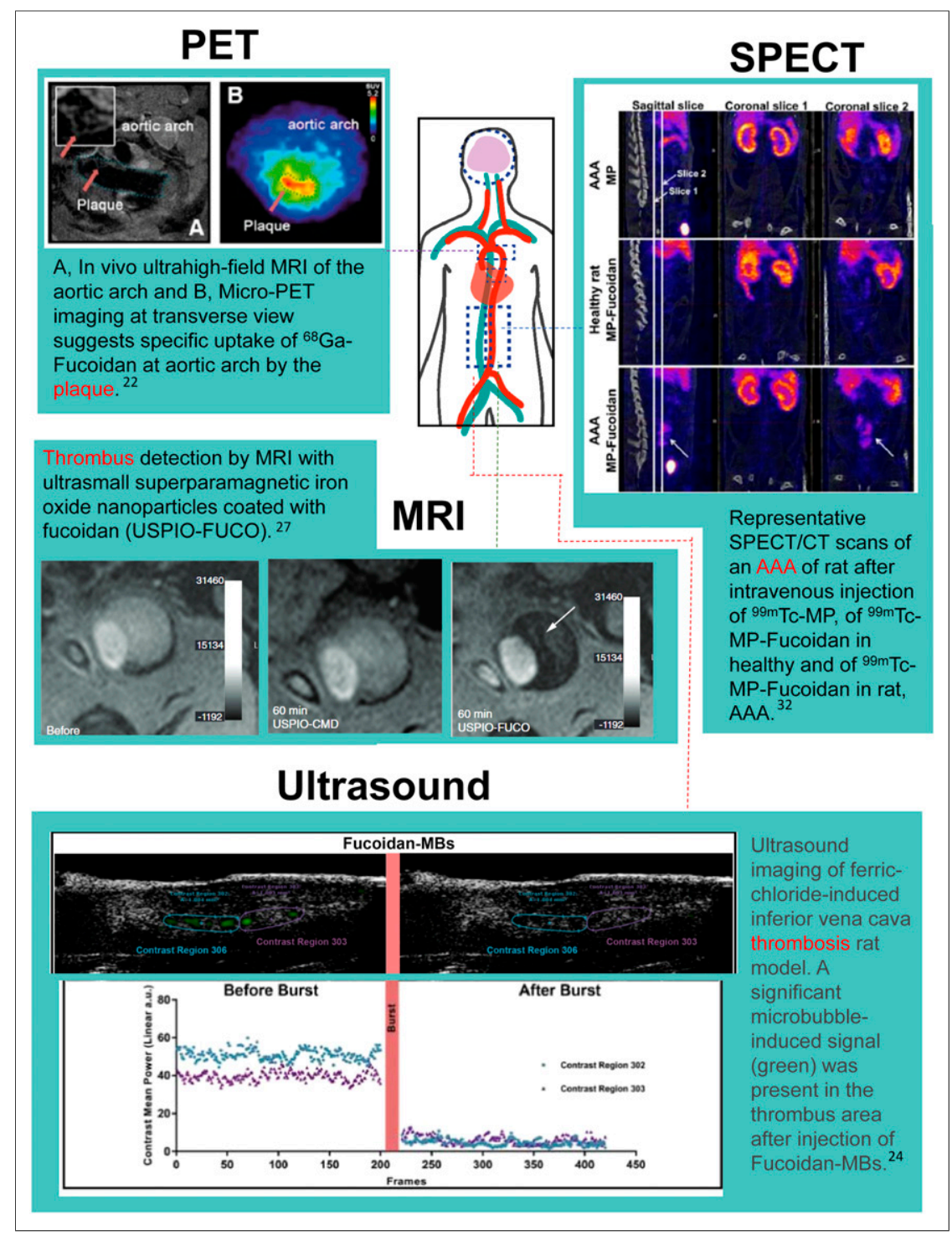

FIGURE 3. (PET) Representative PET and MR images of aortic arch from apolipoprotein E-deficient (apoE-/-) mouse on Western diet for $34 \mathrm{wk}$. Red arrows indicate regions of interest; representation of monitoring P-selectin inhibition by sLeX using ${ }^{68} \mathrm{Ga}-$ fucoidan. MR image was obtained using electrocardiography-triggered multislice, multispin-echo sequence. (SPECT) Coronal slice 1 was centered on abdominal aorta and coronal slice 2 on AAA. Important uptake in AAA was noticed only after injection of 99mTc-MP-fucoidan in sagittal slice and coronal slice 2 (arrows) (32). (MRI) Ultrasmall superparamagnetic iron oxide (USPIO)-FUCO but not USPIO-CMD (control) can detect thrombus. Large intramural thrombus was analyzed in vivo by MRI before or $1 \mathrm{~h}$ after injection of USPIO-CMD or USPIO-FUCO. Strong hyposignal in aorta (arrow) was observed only after USPIO-FUCO injection. MRI signal is evaluated in gray scale reported in arbitrary units (27). (Ultrasound) Images were analyzed for $10 \mathrm{~min}$ after injection of microbubbles (MBs). Fifty frames after destructive pulse were selected as reference, and contrast signals seen in frame only before burst were highlighted in green. Significant microbubble-induced signal (green) was present in thrombus area after injection of fucoidan-microbubbles (24).

overexpressed in cardiovascular disease. The polysaccharidecoated bubbles were developed by a new, simple 1-step process and were shown to target $\mathrm{P}$-selectin in vitro using flow chambers with activated platelets (23). The fucoidan microbubbles were used in an $\mathrm{FeCl}_{3}$-induced thrombus rat model, showing localization to the thrombotic area (Fig. 3) (24). The approach demonstrated a straightforward methodology that resulted in specific thrombus detection. Furthermore, it could lend itself to therapeutic applications by using P-selectin-targeted microbubbles as vectors for drugs. A recent study by the same group investigated a thrombolytic therapy based on fucoidan-functionalized polymer nanoparticles loaded with drug for thrombolysis (25).

Atrial fibrillation is the most common cardiac arrhythmia and is associated with a high thrombotic risk, with thrombus formation commonly occurring in the left atrium. Jing et al. investigated an ultrasound imaging approach using anti-P-selectin antibody-labeled microbubbles to evaluate the risk of thrombus formation in the left atrium of a rat atrial fibrillation model (21). The study showed that Pselectin-targeted microbubbles had high labeling specificity and detected $\mathrm{P}$-selectin expression in the left atrium of rats. The results suggest that this molecular imaging approach could be used to measure the thrombotic risk of patients in atrial fibrillation based on the P-selectin levels in the left atrium.

MRI is a widely used modality for detecting and visualizing the morphologic characteristics of atherothrombosis, but higher resolution is needed to identify earlier signs of disease. Preclinical studies have aimed to develop MRI contrast agents targeted to Pselectin for enhancing the detection of early thrombus.

A study by Suzuki et al. described ultrasmall superparamagnetic iron oxide nanoparticles coated with fucoidan for MRI of thrombi in an abdominal aortic aneurysm (AAA) rat model in which intraluminal thrombus forms at the area of aneurysm (27). This research applied the fucoidan P-selectintargeting concept of their previously reported SPECT imaging approach, which used ${ }^{99 \mathrm{~m}} \mathrm{Tc}$-fucoidan to detect intravascular thrombi in vivo (26). In the MRI study, Suzuki et al. analyzed a large intramural thrombus before and after injection with fucoidan-conjugated ultrasmall superparamagnetic iron oxide and found that the intraluminal signal measured by MRI 
corresponded to histologically confirmed thrombi. Significant signal attenuation at the thrombus site occurred 15 min after injection, with the signal maximizing between 1 and $2 \mathrm{~h}$ (Fig. 3 ) and returning to baseline after $4 \mathrm{~h}$. The results showed the feasibility of fucoidan labeling of P-selectin in thrombi.

A recent publication also reported on an MRI approach for imaging atherothrombosis in a aneurysmal thrombosis rat model using fucoidan-coated ultrasmall superparamagnetic and luminescent $\mathrm{Zn}(\mathrm{Fe}) \mathrm{O}$ hybrid nanoparticles. These nanoparticles contain an iron-to-zinc ratio of 0.50 , which offers bimodal versatility for both MRI and optical imaging capabilities (28). The study by Nguyen et al. showed increased MRI signal within 30-60 min of nanoparticle injection in a rat with a confirmed small thrombus, with the maximum signal reached after $2 \mathrm{~h}$. MRI of injected nanoparticles identified diseased tissue, which was then harvested and imaged with an optical microscope to assess nanoparticle luminescence. Although there was large optical imaging autofluorescence associated with the collagen in the vessel wall, bright regions in the thrombus could represent high amounts of the P-selectin-targeted nanoparticles (28).

Both MRI studies used fucoidan as a P-selectin-targeting reagent in their nanoparticle formulations with promising results, demonstrating the value of P-selectin imaging for enhancing early detection of thrombus formation.

To help improve the early detection of acute ischemic stroke, Israel et al. imaged the course of P-selectin expression in a rat stroke model. The same group previously developed a P-selectin-targeted PET radiotracer, fucoidan labeled with ${ }^{68} \mathrm{Ga}$, for imaging vulnerable atherosclerotic plaques (described above) (29). Using ${ }^{68} \mathrm{Ga}$-fucoidan, the authors performed PET imaging of P-selectin after inducing photothrombotic stroke in rats; acquired images at 2-5, 24-26, and $48 \mathrm{~h}$ after ischemic stroke; and found that at the $2-5 \mathrm{~h}$ and $48 \mathrm{~h}$ after stroke time point there was significantly increased ${ }^{68} \mathrm{Ga}$-fucoidan signal in the stroke lesion compared with the contralateral side. The tracer signal decreased significantly after $24 \mathrm{~h}$ and was no longer elevated at $48 \mathrm{~h}$. $\mathrm{P}$-selectin PET imaging may provide a valuable tool to evaluate the early stage of acute ischemic stroke, when appropriate therapy can be applied within the critical treatment time frame.

P-selectin is also implicated in early AAA development (30). Similar to the other cardiovascular diseases discussed, there is a lack of reliable early detection methods, and there are challenges in predicting the risk of rupture, an oftenfatal complication. Endothelial activation in initial AAA development could be a beneficial progression biomarker. Inflammation in the abdominal aorta of a tumor necrosis factor $\alpha$-exposed C57BL/6 mouse has been detected with ultrasound imaging of P-selectin-targeted microbubbles (31). Another approach to the early detection of AAA used ${ }^{99 \mathrm{~m} T c-}$ radiolabeled microparticles functionalized with fucoidan for SPECT imaging (32). The study reported a strong-signal uptake in the aneurysm 30 min after intravenous injection in a rat AAA model (Fig. 3). Specifically, the SPECT signal was 3 times higher in the aortic aneurysm than in the renal aorta internal control and was absent in control rats. Interestingly, the authors developed 2 different formulations of tracers that exhibited different uptake results. ${ }^{99 \mathrm{~m}} \mathrm{Tc}$ radiolabeled to fucoidan without a microparticle showed uptake only in the AAA intraluminal thrombus region, whereas 99mTc-microparticle-fucoidan demonstrated increased binding capability by labeling the entire aneurysm. This novel ${ }^{99 \mathrm{~m} T c}$-labeled fucoidan-conjugated microparticle targeting P-selectin displayed specific AAA uptake and could serve as a beneficial diagnostic tool for AAA; more testing will reveal its capability in detecting the initial stages of AAA (32).

\section{P-SELECTIN AS A THERAPEUTIC TARGET: CLINICAL STUDIES}

The evidence reviewed in the prior sections shows that $\mathrm{P}$ selectin is heavily implicated in the early development and progression of different inflammatory diseases and that it could be harnessed as an imaging biomarker. Another line of research has investigated its potential use as a therapeutic target. In particular, recent studies on patients have explored the role of P-selectin targeting in myocardial infarction and sickle cell disease.

Crizanlizumab is the first P-selectin-targeting drug nearing Food and Drug Administration approval, with the initial indication being for targeting vasoocclusive crises in sickle cell disease patients. Exciting results from the phase II SUSTAIN clinical trial in sickle cell disease demonstrated that crizanlizumab reduced the median rate of painful vasoocclusive events, which involve an aggregation of leukocytes and red blood cells creating a painful blockage in small blood vessels $(33,34)$. Blocking elevated expression of P-selectin in sickle cell disease can help prevent the adherence of leukocytes and red blood cells within the postcapillary venules. Therapeutic targeting of P-selectin would benefit from being able to image P-selectin expression levels and identify high-signal locations for treatment planning. Additionally, the small-molecule pan-selectin inhibitor rivipansel has been investigated in sickle cell disease, although it has a greater inhibitory effect on E-selectin than on P-selectin (35). Based on initial clinical evidence, there was hope that rivipansel would abort vasoocclusive crises when given acutely in hospitalized patients. However, rivipansel did not meet the efficacy goals in a phase III clinical trial, a result that underscores the challenges of reversing acute inflammation and vasoocclusion in sickle cell disease (36). Thus, prevention of vasoocclusion by way of prophylactic antiadhesion treatments (e.g., crizanlizumab) remains the only viable therapeutic strategy for sickle cell disease, at present.

Another P-selectin-blocking antibody, inclacumab, significantly reduced myocardial damage (measured by troponin I and creatine kinase levels) after percutaneous coronary intervention for non-ST-segment elevation myocardial infarction, with the best results being obtained when 
TABLE 1

Molecular Imaging of P-Selectin in Cardiovascular Diseases

\begin{tabular}{|c|c|c|c|}
\hline Publication focus & Imaging modality & Agent & Reference \\
\hline Thrombotic risk & Ultrasound & Anti-P-selectin microbubbles & (21) \\
\hline Vulnerable plaques & PET & ${ }^{68} \mathrm{Ga}$-fucoidan & (22) \\
\hline Cardiovascular inflammation/thrombus & Ultrasound & Fucoidan microbubbles & $(23,24)$ \\
\hline Platelet-rich thrombus/endothelial activation & SPECT & 99mTc-fucoidan & (26) \\
\hline Intraluminal thrombus & MRI & USPIO-fucoidan nanoparticles & $(27)$ \\
\hline Atherothrombosis & MRI & $\mathrm{Zn}(\mathrm{Fe}) \mathrm{O}$-fucoidan nanoparticles & $(28)$ \\
\hline Thrombotic stroke & PET & ${ }^{68} \mathrm{Ga}$-fucoidan & (29) \\
\hline Inflammation in abdominal aorta & Ultrasound & Anti-P-selectin microbubbles & (31) \\
\hline Abdominal aortic aneurysm & SPECT & 99mTc-fucoidan & (32) \\
\hline
\end{tabular}

treatment was administered less than $3 \mathrm{~h}$ before percutaneous coronary intervention (37). P-selectin imaging before the procedure might help assess the myocardial infarction and determine the blocking strategy to reduce further damage.

In summary, P-selectin-targeted biologicals are intensely investigated in preclinical and clinical studies, and we anticipate that other targeted treatments will be developed on the heels of the studies with crizanlizumab, rivipansel, and inclacumab.

\section{SUMMARY}

Within the past 5 years, there have been several reported studies of molecular imaging agents for imaging P-selectin in inflammatory conditions, particularly cardiovascular diseases, for which there is a lack of imaging tools for early diagnosis. Preclinical molecular imaging of P-selectin has been demonstrated across PET, SPECT, MRI, and ultrasound techniques with successful results using various agents functionalized with fucoidan or anti-P-selectin antibody (Table 1). As Bonnard et al. demonstrated, creating molecular imaging agents, with or without microparticle or nanoparticle carriers, can influence P-selectin binding capabilities and ultimately enhance detection sensitivity, underscoring how imaging agent development plays a crucial part in each study (38).

In addition, different $\mathrm{P}$-selectin imaging strategies and imaging modality characteristics may best be designed toward a specific feature in the inflammatory condition, such as thrombus, plaque, aneurysm, or ischemic stroke. Thus, Pselectin imaging may be a versatile tool, as expression levels are heavily correlated to different stages of inflammatory processes. The direct and early assessment of disease-specific endothelial activation, dysfunction, or platelet activation could help determine the best treatment strategy for each patient. Targeting P-selectin over other adhesion molecules offers the advantage of visualizing inflammation earlier, as P-selectin is the first adhesion molecule expressed by activated endothelium. However, a soluble form of P-selectin can be found in plasma, which could potentially cause an elevated background signal, underscoring the importance of developing sensitive imaging strategies with a high signal-to-noise ratio, especially during the initial stages of inflammation.

Preclinical molecular imaging of P-selectin has been shown to be robust across molecular imaging techniques and shows feasibility as a biomarker of inflammation in cardiovascular disease states. A recent abstract reports the first-in-humans evaluation (phase I) of SPECT ${ }^{99 \mathrm{~m} T c-l a b e l e d}$ fucoidan for P-selectin imaging, demonstrating a good biodistribution and safety profile (39). This is an encouraging first step toward having a P-selectin-targeted imaging agent for unstable plaques and thrombosis in patients.

\section{DISCLOSURE}

Lydia Perkins is supported by the NIH training grant "Training Program in Imaging Sciences in Translational Cardiovascular Research" (T32 HL129964), and funding is also provided by the Pittsburgh Foundation. No other potential conflict of interest relevant to this article was reported.

\section{REFERENCES}

1. Liu CH, Abrams ND, Carrick DM, et al. Biomarkers of chronic inflammation in disease development and prevention: challenges and opportunities. Nat Immunol. 2017;18:1175-1180.

2. Lee HJ, Ehlerding EB, Cai W. Antibody-based tracers for PET/SPECT imaging of chronic inflammatory diseases. ChemBioChem. 2019;20:422-436.

3. Hammoud DA. Molecular imaging of inflammation: current status. J Nucl Med. 2016;57:1161-1165.

4. Autio A, Jalkanen S, Roivainen A. Nuclear imaging of inflammation: homingassociated molecules as targets. EJNMMI Res. 2013;3:1.

5. Roivainen A, Li X-G, Nanni C, Cai W, Ohrndorf S. In vivo imaging of inflammation and infection. Contrast Media Mol Imaging. 2018;2018:3817871.

6. Gauberti M, Fournier AP, Docagne F, Vivien D, Martinez de Lizarrondo S. Molecular magnetic resonance imaging of endothelial activation in the central nervous system. Theranostics. 2018;8:1195-1212.

7. Abou-Elkacem L, Bachawal SV, Willmann JK. Ultrasound molecular imaging: moving toward clinical translation. Eur J Radiol. 2015;84:1685-1693.

8. Granger DN, Senchenkova E. Leukocyte-endothelial cell adhesion. In: Inflammation and the Microcirculation. San Rafael, CA: Morgan and Claypool; 2010:29-40. 
9. Steyers CM, Miller FJ. Endothelial dysfunction in chronic inflammatory diseases. Int J Mol Sci. 2014;15:11324-11349.

10. Thomas MR, Storey RF. The role of platelets in inflammation. Thromb Haemost. 2015;114:449-458.

11. Yun S-H, Sim E-H, Goh R-Y, Park J-I, Han J-Y. Platelet activation: the mechanisms and potential biomarkers. BioMed Res Int. 2016;2016:9060143.

12. Kappelmayer J, Nagy B. The interaction of selectins and PSGL-1 as a key component in thrombus formation and cancer progression. BioMed Res Int. 2017;2017: 6138145.

13. Silva AKA, Letourneur D, Chauvierre C. Polysaccharide nanosystems for future progress in cardiovascular pathologies. Theranostics. 2014;4:579-591.

14. Wang Y, Xing M, Cao Q, Ji A, Liang H, Song S. Biological activities of fucoidan and the factors mediating its therapeutic effects: a review of recent studies. Mar Drugs. 2019;17:E183.

15. Stewart J, Manmathan G, Wilkinson P. Primary prevention of cardiovascular disease: a review of contemporary guidance and literature. JRSM Cardiovasc Dis. 2017;6:2048004016687211.

16. Cardiovascular diseases: data and statistics. World Health Organization-Europe website. http://www.euro.who.int/en/health-topics/noncommunicable-diseases/ cardiovascular-diseases/data-and-statistics. Accessed October 15, 2019.

17. Tarkin JM, Dweck MR, Evans NR, et al. Imaging atherosclerosis. Circ Res. 2016;118:750-769.

18. Groenendyk JW, Mehta NN. Applying the ordinal model of atherosclerosis to imaging science: a brief review. Open Heart. 2018;5:e000861.

19. Rafieian-Kopaei M, Setorki M, Doudi M, Baradaran A, Nasri H. Atherosclerosis: process, indicators, risk factors and new hopes. Int J Prev Med. 2014;5:927-946.

20. Tabas I, García-Cardeña G, Owens GK. Recent insights into the cellular biology of atherosclerosis. J Cell Biol. 2015;209:13-22.

21. Jing $\mathrm{Y}, \mathrm{Hu} \mathrm{Y}, \mathrm{Li} \mathrm{H}$, et al. Assessment of thrombotic risk in atrial fibrillation with ultrasound molecular imaging of P-selectin. Thromb Haemost. 2018;118:388-400.

22. Li X, Bauer W, Israel I, et al. Targeting P-selectin by gallium-68-labeled fucoidan positron emission tomography for noninvasive characterization of vulnerable plaques: correlation with in vivo 17.6T MRI. Arterioscler Thromb Vasc Biol. 2014;34:1661-1667.

23. Li B, Juenet M, Aid-Launais R, et al. Development of polymer microcapsules functionalized with fucoidan to target $\mathrm{P}$-selectin overexpressed in cardiovascular diseases. Adv Healthc Mater. 2017;6.

24. Li B, Aid-Launais R, Labour M-N, et al. Functionalized polymer microbubbles as new molecular ultrasound contrast agent to target P-selectin in thrombus. Biomaterials. 2019;194:139-150.

25. Juenet M, Aid-Launais R, Li B, et al. Thrombolytic therapy based on fucoidanfunctionalized polymer nanoparticles targeting P-selectin. Biomaterials. 2018;156: 204-216.
26. Rouzet F, Bachelet-Violette L, Alsac J-M, et al. Radiolabeled fucoidan as a p-selectin targeting agent for in vivo imaging of platelet-rich thrombus and endothelial activation. J Nucl Med. 2011;52:1433-1440.

27. Suzuki M, Bachelet-Violette L, Rouzet F, et al. Ultrasmall superparamagnetic iron oxide nanoparticles coated with fucoidan for molecular MRI of intraluminal thrombus. Nanomedicine (Lond). 2015;10:73-87.

28. Nguyen H, Tinet E, Chauveau T, et al. Bimodal fucoidan-coated zinc oxide/iron oxidebased nanoparticles for the imaging of atherothrombosis. Molecules. 2019;24:E962.

29. Israel I, Fluri F, Schadt F, Buck AK, Samnick S. Positron emission tomography and autoradiography imaging of P-selectin activation using ${ }^{68} \mathrm{Ga}$-fucoidan in photothrombotic stroke. Curr Neurovasc Res. 2018;15:55-62.

30. Zhou M, Ding Y, Cai L, Wang Y, Lin C, Shi Z. Low molecular weight fucoidan attenuates experimental abdominal aortic aneurysm through interfering the leukocyte-endothelial cells interaction. Mol Med Rep. 2018;17:7089-7096.

31. Wang S, Unnikrishnan S, Herbst EB, Klibanov AL, Mauldin FW, Hossack JA. Ultrasound molecular imaging of inflammation in mouse abdominal aorta. Invest Radiol. 2017;52:499-506.

32. Bonnard T, Yang G, Petiet A, et al. Abdominal aortic aneurysms targeted by functionalized polysaccharide microparticles: a new tool for SPECT imaging. Theranostics. 2014;4:592-603.

33. Ataga KI, Kutlar A, Kanter J, et al. Crizanlizumab for the prevention of pain crises in sickle cell disease. N Engl J Med. 2017;376:429-439.

34. Novartis announces new crizanlizumab (SEG101) data analysis in sickle cell disease, and investment in SENTRY clinical program. Novartis website. https:// www.pharma.us.novartis.com/news/media-releases/novartis-announces-newcrizanlizumab-seg101-data-analysis-sickle-cell-disease. Published December 1, 2018. Accessed October 15, 2019.

35. Jasuja R, Suidan G, Hett SP, et al. Rivipansel: a small pan-selectin antagonist improves cerebral perfusion and inhibits leukocyte adhesion and in murine sickle cell disease model. Blood. 2016;128:270.

36. Pfizer announces phase 3 top-line results for rivipansel in patients with sickle cell disease experiencing a vaso-occlusive crisis. Pfizer website. https://www.pfizer. com/news/press-release/press-release-detail/pfizer_announces_phase_3_top_line_ results_for_rivipansel_in_patients_with_sickle_cell_disease_experiencing_a_vaso_ occlusive_crisis. Published August 2, 2019. Accessed October 15, 2019.

37. Stähli BE, Gebhard C, Duchatelle V, et al. Effects of the P-selectin antagonist inclacumab on myocardial damage after percutaneous coronary intervention according to timing of infusion: insights from the SELECT-ACS trial. J Am Heart Assoc. 2016;5:e04255.

38. Bonnard T, Serfaty J-M, Journé C, et al. Leukocyte mimetic polysaccharide microparticles tracked in vivo on activated endothelium and in abdominal aortic aneurysm. Acta Biomater. 2014;10:3535-3545.

39. Zheng KH, Kaiser Y, Poel E, et al. ${ }^{99 m} \mathrm{Tc}$-fucoidan as diagnostic agent for P-selectin imaging: first-in-human evaluation (phase I) [abstract]. Atherosclerosis. 2019;287:e143. 